\title{
SECURING THE AIRWAY PATENCY BY FIREFIGHTERS WITH THE USE OF COMBITUBE. A PILOT DATA
}

\author{
Michael Frass ${ }^{1}$, Oliver Robak ${ }^{1}$, Jacek Smereka ${ }^{2}$, Agata Dabrowska ${ }^{3}$, Marek Dabrowski ${ }^{3}$, \\ Michael Czekajlo ${ }^{4}$, Wladyslaw Gawel ${ }^{5}$, Lukasz Szarpak ${ }^{6}$ \\ 'Department of Internal Medicine I, Medical University of Vienna, Vienna, Austria \\ ${ }^{2}$ Department of Emergency Medical Service, Wroclaw Medical University, Wroclaw, Poland \\ ${ }^{3}$ Department of Rescue Medicine, Poznan University of Medical Sciences, Poznan, Poland \\ ${ }^{4}$ Hunter Holmes McGuire VA Medical Centre, Department of Surgery, Richmond, United States \\ ${ }^{5}$ Student's Scientific Association of Children's Diabetology of Medical University of Silesia, Katowice, Poland \\ ${ }^{6}$ Lazarski University, Warsaw, Poland
}

\begin{abstract}
INTRODUCTION: The protection of the airways with the use of vomer devices for ventilation is one of the elements of the procedure in cardiopulmonary resuscitation. One of the alternative ways to protect the airways from endotracheal intubation is the CombiTube tube. The aim of the study was to assess the ability to protect airway patency using CombiTube during simulated cardiopulmonary resuscitation performed by firefighters.
\end{abstract}

METHOD: This study was a prospective randomized crossover simulation study. The study included 56 firefighters who performed airway patency protection with the use of CombiTube during simulated cardiopulmonary resuscitation with and without chest compressions.

RESULTS: The median duration of securing airway patency with CombiTube device during scenario without and with chest compressions was: $21 \mathrm{~s}(\mathrm{IQR} ; 14-25.5)$ vs. $21.5 \mathrm{~s}$ (IQR; 15-27), respectively. The vast majority of attempts to insert CombiTube resulted in the insertion of the device to the esophagus: $92.8 \%$ vs. $91.1 \%$ (with and without chest compressions, respectively). The insertion of CombiTube to the trachea was observed in $7.1 \%$ vs. $8.9 \%$ during scenarios with and without chest compression, respectively. Study participants assessed the easiness of performing the procedure at 19 points (IQR, 13-22) for scenario without chest compression, and 18.5 points (IQR, 14-21.5) for scenario with uninterrupted chest compressions.

CONCLUSIONS: Firefighters are able to secure the airway patency with the use of CombiTube tube after a short training. Compressing the chest during resuscitation does not prolong the procedure of maintaining the airway patency with CombiTube. The tip of the CombiTube tube is inserted into the esophagus in over $91 \%$ of cases.

KEY WORDS: airway management, firefighter, prehospital, cardiopulmonary resuscitation, medical simulation

Disaster Emerg Med J 2018; 3(2): 46-50 


\section{INTRODUCTION}

Securing the airways patency in prehospital conditions may be a challenge for paramedics [1]. The endotracheal intubation still remains the golden standard, however, the use of it is limited by the necessity of having experienced medical personnel [2-4]. When performing the rescuing procedures by firefighters-paramedics the use of supraglottic airway devices (SADs) is a more appropriate method.

The European Resuscitation Council (ERC), similarly to the American Heart Association (AHA) in their updated guidelines for cardiopulmonary resuscitation, sanctioned the use of supraglottic airway devices for ventilation during cardiopulmonary resuscitation [5]. Thanks to this update, it is now possible to perform asynchronous resuscitation and ventilation when using SADs.

The CombiTube tube (Sheridan Catheter Corporation, Argyle, NY, USA) is an example of airway patency securing device. The CombiTube tube is a double-

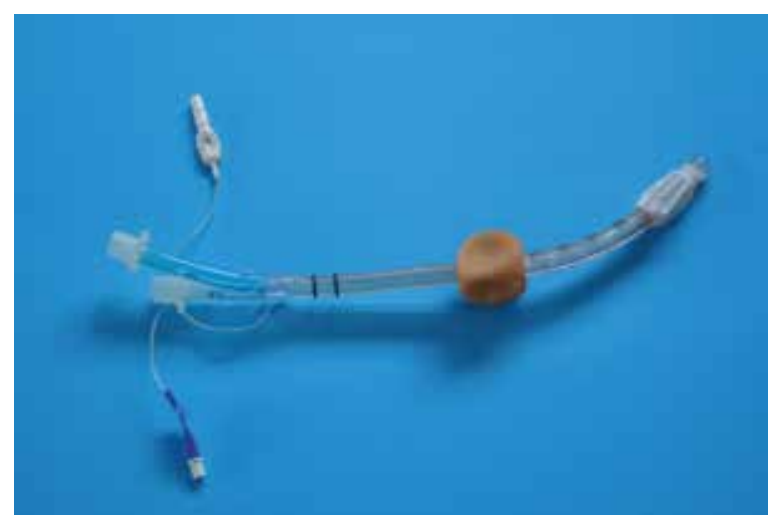

FIGURE 1. CombiTube airway device

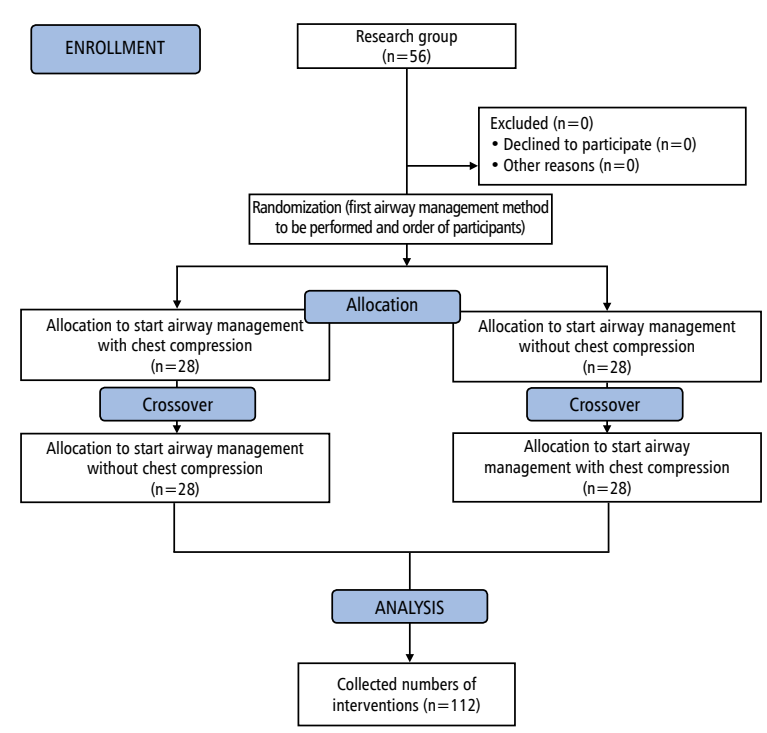

FIGURE 2. Consort Flow diagram -lumen tube and thanks to its construction it maintains its function when inserted both into the esophagus or, as it happens less often, in the trachea $[6,7]$. The longer channel is coloured in blue and its distal end is blind, however the device has holes on the side which allows for ventilation from the side of the throat - similarly to the laryngeal tube (LTD). The second air channel does not have holes on its side but has an opening on its distal end, so that when it is inserted into the trachea, it can work as the tracheal tube [8]. The CombiTube device is secured with two sealing cuffs: distal (esophagus) and significantly larger proximal (oral-pharyngeal). CombiTube tubes are available in only two sizes: 42 French and 37 French.

The aim of the study was to assess the ability to secure airway patency with the use of CombiTube performed by firefighters during simulated cardiopulmonary resuscitation.

\section{METHOD}

The study was approved by the Institutional Review Board of the Polish Society of Disaster Medicine (Approval no. 31.02.2018.IRB) and was conducted as a prospective, randomized crossover simulation study. This study is a continuation of the series of the authorial studies regarding the most effective way of securing the airway patency in prehospital conditions [9-12].

The study inclusion criterion was being an active firefighter working for the State Fire Service. The exclusion criteria included: back or hand pain that didn't allow for performing cardio-pulmonary resuscitation. Moreover, voluntary written informed consent was obtained from each participant before study inclusion. The study included 56 firefighters. None of the participants had received CombiTube training before the study.

During the study participants were given instruction on the proper ventilation technique with the use of supraglottic airway devices. Then the participants of the study had participated in a 10-minute practical session.

One week after the study, participants were asked to secure airway patency with the use of CombiTube during simulated cardiopulmonary resuscitation in the scenarios with and without chest compressions. The order of research scenarios and participants was randomized with the coin tossing method. A detailed randomization procedure for the study is shown on Figure 2. In order to unify the 


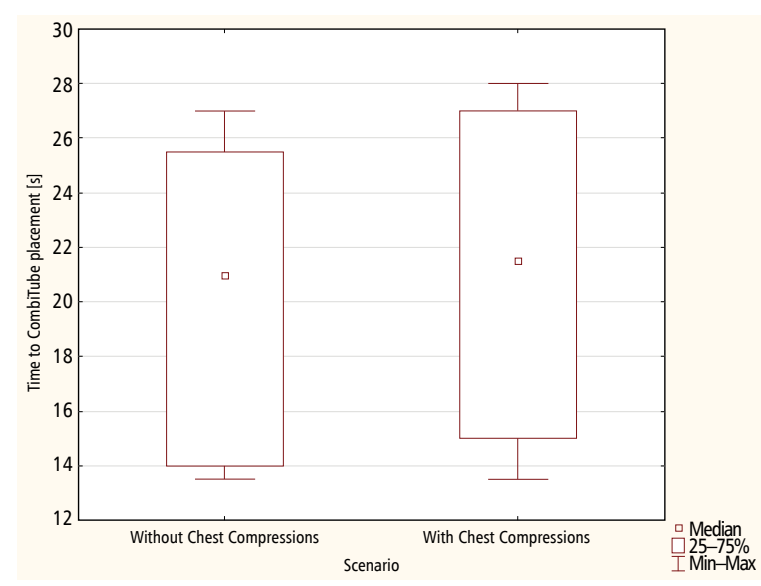

FIGURE 3. Median time to airway management using CombiTube

difficulties resulting from chest compressions, the LUCAS3 chest compression system was used.

The main parameter measured in the study was the time of securing airway patency with the use of CombiTube. It was defined as the time from taking the device into the hand until securing the device with two sealing cuffs and attempting to ventilate with a self-expanding bag. It was additionally assessed whether the CombiTube tube was inserted into the esophagus or trachea. In addition, the easiness of performing this procedure was measured using a 100-degree scale $(1-$ the procedure is easy to perform, 100 - the procedure is extremely difficult to perform).

Statistica 13.1EN software (Tibco Inc., Tulsa, OK, USA) was used for statistical analysis. Categorical data are described by absolute numbers and percentages. Continuous data were described by median and interquartile range (IQR). All values are two-sided and $p<0.05$ was considered to be statistically significant.

\section{RESULTS}

The study included 56 firefighters-paramedics with median age - 33.5 (IQR; 29-36) years, and median work experience - 9.4 (IQR; 6-12.5) years.

The median time of securing the airways with the use of CombiTube during both research scenarios varied and amounted for $21 \mathrm{~s}$ (IQR: 14-25.5) and $21.5 \mathrm{~s}$ (IQR: 15-27) respectively for scenarios with and without continuous chest compression (Fig. 3).

The vast majority of the CombiTube insertion attempts ended up in the insertion into the esophagus. It happened in both scenarios i.e. without chest compressions as well as with continuous chest compression, amounting for $91.1 \%$ and $92.8 \%$ respectively. The insertion of CombiTube into the trachea was observed in $7.1 \%$ vs. $8.9 \%$ for the scenario with and without continuous chest compressions, respectively (Tab. 1).

The study participant assessed the easiness of performing the procedure for 19 points (IQR; 13-22) in the scenario without chest compression vs. 18.5 points (IQR; 14-21.5) for scenario with uninterrupted chest compressions.

\section{DISCUSSION}

Securing the airway patency is one of the most basic elements when dealing with the patients with an airways obstruction or lack of breath. In the conducted study we have presented that firefighters-paramedics just after a short training are able to use the CombiTube tube to secure the patient's airways during simulated cardiopulmonary resuscitation in the scenarios with and without chest compressions. Other authors [13-16] have also proven the easiness of using CombiTube.

Securing airway patency with the use of different devices such as supraglottic airway devices or

\begin{tabular}{|l|l|l|l|}
\hline \multicolumn{1}{|c|}{ Table 1. The results of the study } & \multicolumn{1}{|c|}{$\begin{array}{c}\text { Scenario without chest } \\
\text { compressions }\end{array}$} & $\begin{array}{l}\text { Scenario with uninterrupted } \\
\text { chest compressions }\end{array}$ & p-value \\
\hline Time to Combitube placement & $\begin{array}{l}21 \\
(I Q R ; 14-25.5)\end{array}$ & $\begin{array}{l}21.5 \\
(I Q R ; 15-27)\end{array}$ & NS \\
\hline $\begin{array}{l}\text { Insertion of the tube into the } \\
\text { esophagus }\end{array}$ & $51(91.1 \%)$ & $52(92.8 \%)$ & NS \\
\hline $\begin{array}{l}\text { Insertion of the tube into the } \\
\text { trachea }\end{array}$ & $5(8.9 \%)$ & $4(7.1 \%)$ & NS \\
\hline $\begin{array}{l}\text { Easiness of performing the } \\
\text { procedure }\end{array}$ & $\begin{array}{l}19 \\
(I Q R ; 13-22)\end{array}$ & $\begin{array}{l}18.5 \\
(I Q R ; 14-21.5)\end{array}$ & \\
\hline
\end{tabular}

${ }^{*} \mathrm{NS}=$ not statistically significant. 
endotracheal tube allows for asynchronous resuscitation, which results with optimization of patient's oxygenation and reduces the breaks in chest compressions, both of which increase the chances of spontaneous circulation return. An additional aspect in favour of using this type of devices during cardiopulmonary resuscitation is the fact that, as indicated by numerous studies $[12,17,18]$, when performing the endotracheal intubation, the compression of the chest significantly reduces the effectiveness of the first intubation attempt and prolongs the time of the procedure, however, this is not a problem when using supraglottic airway devices, including CombiTube.

The CombiTube is a device which combines the benefits of laryngeal tube and intubation tube $[19,20]$. In his study Rumball et al. [21] compared the easiness and effectiveness of patient ventilation with the use of CombiTube, laryngeal mask airway and the oral-pharyngeal tube. In that study the CombiTube turned out to be the best in both effectiveness of insertion and ventilation, and its use was the most preferred in the paramedics included in the study. Due to the numerous studies, which indicated the benefits of using CombiTube, Bollig recommends the CombiTube in the Scandinavian guidelines for prehospital airway management [22]. As Rabitsch et al. indicated the CombiTube worked well in cases of difficult access to the patient's head and in bleeding and vomiting patients [23].

It is worth noting that the CombiTube beside its undoubted benefits in its use in emergency medicine has its use also in the conditions of the operating theatre $[8,13,24]$.

\section{CONCLUSIONS}

In the conducted simulation study, just after a short training, firefighters-paramedics are able to secure the airway with the use of the CombiTube tube. Compression of the chest during resuscitation does not prolong the time of securing airway when using CombiTube. The tip of the CombiTube tube is inserted into the esophagus in over $91 \%$ of cases.

\section{REFERENCES}

1. Qureshi MJ, Kumar M. Laryngeal mask airway versus bag-mask ventilation or endotracheal intubation for neonatal resuscitation. Cochrane Database Syst Rev. 2018; 3: CD003314, doi: 10.1002/14651858. CD003314.pub3, indexed in Pubmed: 29542112.

2. Moors XRJ, Rijs K, Den Hartog D, et al. Pediatric out-of-hospital cardiopulmonary resuscitation by helicopter emergency medical service, does it has added value compared to regular emergency medical service? Eur J Trauma Emerg Surg. 2018; 44(3): 407-410, doi: 10.1007/s00068-017-0815-5, indexed in Pubmed: 28711949.

3. Kurowski A, Szarpak L, Zasko P, et al. Comparison of direct intubation and Supraglottic Airway Laryngopharyngeal Tube (S.A.L.T.) for endotracheal intubation during cardiopulmonary resuscitation. Randomized manikin study. Anaesthesiol Intensive Ther. 2015; 47(3): 195-199, doi: 10.5603/AlT.2015.0031, indexed in Pubmed: 26165236.

4. Benoit JL, Gerecht RB, Steuerwald MT, et al. Endotracheal intubation versus supraglottic airway placement in out-of-hospital cardiac arrest: A meta-analysis. Resuscitation. 2015; 93: 20-26, doi: 10.1016/j. resuscitation.2015.05.007, indexed in Pubmed: 26006743.

5. Kleinman ME, Goldberger ZD, Rea T, et al. 2017 American Heart Association Focused Update on Adult Basic Life Support and Cardiopulmonary Resuscitation Quality: An Update to the American Heart Association Guidelines for Cardiopulmonary Resuscitation and Emergency Cardiovascular Care. Circulation. 2018; 137(1): e7-ee13, doi: 10.1161/CIR.0000000000000539, indexed in Pubmed: 29114008.

6. Gaitini LA, Vaida SJ, Agro F. The Esophageal-Tracheal Combitube. Anesthesiol Clin North America. 2002; 20(4): 893-906, indexed in Pubmed: 12512268.

7. An J, Nam SB, Lee JS, et al. Comparison of the i-gel and other supraglottic airways in adult manikin studies: Systematic review and meta-analysis. Medicine (Baltimore). 2017; 96(1): e5801, doi: 10.1097/ MD.0000000000005801, indexed in Pubmed: 28072732.

8. Choi YJi, Park S, Chi Sl, et al. Combitube insertion in the situation of acute airway obstruction after extubation in patients underwent two-jaw surgery. J Dent Anesth Pain Med. 2015; 15(4): 235-239, doi: 10.17245/jdapm.2015.15.4.235, indexed in Pubmed: 28879285.

9. Smereka J, Czyzewski L, Szarpak L, et al. Comparison between the TrueView EVO2 PCD and direct laryngoscopy for endotracheal intubation performed by paramedics: Preliminary data. Am J Emerg Med. 2017; 35(5): 789-790, doi: 10.1016/j.ajem.2016.11.063, indexed in Pubmed: 27916466.

10. Ladny JR, Bielski K, Szarpak L, et al. Are nurses able to perform blind intubation? Randomized comparison of I-gel and laryngeal mask airway. Am J Emerg Med. 2017; 35(5): 786-787, doi: 10.1016/j. ajem.2016.11.046, indexed in Pubmed: 27899211.

11. Ladny JR, Sierzantowicz R, Kedziora J, et al. Comparison of direct and optical laryngoscopy during simulated cardiopulmonary resus- 
citation. Am J Emerg Med. 2017; 35(3): 518-519, doi: 10.1016/j. ajem.2016.12.026, indexed in Pubmed: 28089239.

12. Aleksandrowicz S, Czyzewski L, Smereka J, et al. Tracheal intubation with a Macintosh laryngoscope with and without chest compressions, performed by nurses. Am J Emerg Med. 2016; 34(12): 2448-2449, doi: 10.1016/j.ajem.2016.09.016, indexed in Pubmed: 27641245.

13. Sethi AK, Desai M, Tyagi $A$, et al. Comparison of combitube, easy tube and tracheal tube for general anesthesia. J Anaesthesiol Clin Pharmacol. 2014; 30(4): 526-532, doi: 10.4103/0970-9185.142849, indexed in Pubmed: 25425779.

14. Saeedi M, Hajiseyedjavadi H, Seyedhosseini J, et al. Comparison of endotracheal intubation, combitube, and laryngeal mask airway between inexperienced and experienced emergency medical staff: A manikin study. Int J Crit IIIn Inj Sci. 2014; 4(4): 303-308, doi: 10.4103/2229-5151.147533, indexed in Pubmed: 25625062.

15. Atherton GL, Johnson JC. Ability of paramedics to use the Combitube in prehospital cardiac arrest. Ann Emerg Med. 1993; 22(8): 1263-1268, indexed in Pubmed: 8333625.

16. Calkins TR, Miller K, Langdorf MI. Success and complication rates with prehospital placement of an esophageal-tracheal combitube as a rescue airway. Prehosp Disaster Med. 2006; 21(2): 97-100, indexed in Pubmed: 16770999.

17. Szarpak L, Karczewska K, Czyzewski L, et al. Airtraq Laryngoscope Versus the Conventional Macintosh Laryngoscope During Pediatric Intubation Performed by Nurses: A Randomized Crossover Manikin Study With Three Airway Scenarios. Pediatr Emerg Care. 2017; 33(11): 735-739, doi: 10.1097/PEC.0000000000000741, indexed in Pubmed: 27228145.
18. Truszewski Z, Czyzewski L, Smereka J, et al. Ability of paramedics to perform endotracheal intubation during continuous chest compressions: a randomized cadaver study comparing Pentax AWS and Macintosh laryngoscopes. Am J Emerg Med. 2016; 34(9): 1835-1839, doi: 10.1016/j.ajem.2016.06.054, indexed in Pubmed: 27369468.

19. Enlund $M$, Miregard $M$, Wennmalm K. The Combitube for failed intubation--instructions for use. Acta Anaesthesiol Scand. 2001; 45(1): 127-128, indexed in Pubmed: 11152025.

20. Mercer M. The role of the Combitube in airway management. Anaesthesia. 2000; 55(4): 394-395, indexed in Pubmed: 10781136.

21. Rumball CJ, MacDonald D. The PTL, Combitube, laryngeal mask, and oral airway: a randomized prehospital comparative study of ventilatory device effectiveness and cost-effectiveness in 470 cases of cardiorespiratory arrest. Prehosp Emerg Care. 1997; 1(1): 1-10, indexed in Pubmed: 9709312.

22. Bollig G. Combitube and Easytube should be included in the Scandinavian guidelines for pre-hospital airway management. Acta Anaesthesiol Scand. 2009; 53(1): 139-140, doi: 10.1111/j.13996576.2008.01848.x, indexed in Pubmed: 19128329.

23. Rabitsch W, Schellongowski P, Staudinger T, et al. Comparison of a conventional tracheal airway with the Combitube in an urban emergency medical services system run by physicians. Resuscitation. 2003; 57(1): 27-32, indexed in Pubmed: 12668296.

24. Zamora JE, Saha TK. Combitube rescue for Cesarean delivery followed by ninth and twelfth cranial nerve dysfunction. Can J Anaesth. 2008; 55(11): 779-784, doi: 10.1007/BF03016352, indexed in Pubmed: 19138919. 